\title{
A ROBUST SOLUTION PROCEDURE FOR THE RIGOROUS METHODS OF SLICES ${ }^{1}$
}

\author{
Hong Zheng ${ }^{\mathrm{i})}$, Chuang Bing Zhou ${ }^{\mathrm{ii})}$ and Defu LiU ${ }^{\mathrm{iii}}$
}

\begin{abstract}
All rigorous methods of slices, i.e., those satisfying all equilibrium conditions, have numerical problems under some conditions, and the worst is "failure to converge" even if the solution exists. This is considered to be the unique reason that those simplified slice methods, i.e., those satisfying part of equilibrium conditions, are still in use. The purpose of this study is to develop a solution procedure for those rigorous methods such as Morgenstern-Price's method. The procedure is so robust that it is able 1) to detect whether the solutions exist within a specified domain;2) to find out all possible solutions; 3 ) and to trace all branches of equilibrium curves in the domain.
\end{abstract}

Key words: factor of safety, limit equilibrium methods, slope stability, slopes (IGC: E6)

\section{INTRODUCTION}

Although the finite element method is playing an increasingly important role in the stability analysis of slopes (Zheng et al., 2005, 2006), the classical limit equilibrium methods still have the final say in the design of slopes in most projects because they are simpler and require fewer soil parameters. Therefore, the investigation on the limit equilibrium methods is still quite active, for example, Cheng et al. (2008), Zhu et al. (2005), Baker (2005), and Husein et al. (2001).

The slice methods fall into two categories: the nonrigorous methods and the rigorous methods. The nonrigorous methods, also called the simplified methods, are those satisfying part of equilibrium conditions, for example, Fellenius's method, Bishop's modified method, Corps of engineer's method, Lowe and Karafiath's method, etc. They were developed on the date when computers had not been used extensively. The rigorous methods, such as Morgenstern-Price's method, Janbu's generalized method and Sarma's method, satisfy all equilibrium conditions and are usually implemented by means of computers. Duncan (1996) and Abramson et al. (1996) made comprehensive reviews on those commonlyused slice methods.

Due to the widespread use of high-performance microcomputers, nowadays only those rigorous methods should be in service and those non-rigorous methods should have retired for at least 10 years. However, this is not the case. In research reports on slope stability analy- sis, we frequently read such tables where some nonrigorous factors of safety are included. This is because the researcher fails to achieve a proper solution while using the rigorous slice methods.

For presentation simplicity, let us take Spencer's method for instance. The cases are similar with the other rigorous methods. Spencer's method assumes the factor of safety, $F$, and the tangent of inclination of the interslice force, $\lambda$, as the basic unknowns. It is well known that the 2-dimensional Spencer's method always leads to a system of nonlinear equations in $F$ and $\lambda$. From geometrical viewpoint, the two equations in the system define in plane $(F, \lambda)$ two types of curves labelled Type-F and Type-M, which represent the loci of the pairs $(F, \lambda)$ in satisfying static force or moment equilibrium, respectively. Our task is to seek the intersections of the two types of curves.

At present, Newton's method (Cheng and Zhu, 2004) and the numerical-graphical method (Chugh, 1981) are two commonly-used procedures for the above-mentioned system of nonlinear equations. Both methods start with two approximate values for $F$ and $\lambda$ respectively, tacitly assuming that there is only one pair $(F, \lambda)$ as the intersection of one curve of type-F and one curve of type-M. Let us suppose that both types have more than one branch, each having an intersection, just as shown in Fig. 4. In this case, unless the intersections are far enough from each other, Newton's method usually fails to converge. If the numerical-graphical method is used, on the other hand, the right intersection in Fig. 4 might be missed

1 Funded by the National Natural Science Foundation of China (NSFC), Project no. 50779031.

i) Professor, Ph.D, State Key Laboratory of Geomechanics and Geotechnical Engineering, Institute of Rock and Soil Mechanics, Chinese Academy of Sciences, China (hzheng@whrsm.ac.cn).

ii) Professor, Ph.D, School of Civil Engineering, Wuhan University, China.

iii) Professor, Ph.D, China Three Gorges University, Key Laboratory of Geological Hazards on Three Gorges Reservoir Area, Ministry of Education, China.

The manuscript for this paper was received for review on July 23, 2008; approved on March 26, 2009.

Written discussions on this paper should be submitted before March 1, 2010 to the Japanese Geotechnical Society, 4-38-2, Sengoku, Bunkyo$\mathrm{ku}$, Tokyo 112-0011, Japan. Upon request the closing date may be extended one month. 
while an improper intersection might be sought. An improper intersection means that the system of forces resulting from the intersection is not admissible, for example, the normal pressure on some bases of slices is negative.

We should mention that the numerical-graphical method was named by Chugh (1981). The authors would rather use the name: "the decoupled method" according to its solution procedure. Actually, Baker (1980) also proposed a similar method.

Using Spencer's method, Chugh (1981) gave an example where two branches of equilibrium curves are encountered with two intersections. The example will be repeated subsequently. Recently Cheng et al. (2008) listed more examples that have no convergent solutions if conventional procedures are used.

Cheng (2003) observed that the equation derived from the force equilibrium conditions of $n$ slices can be transformed into an $n$-th degree polynomial equation in $F$ with parameter $\lambda$. With an assumed parameter $\lambda$ and using the double QR algorithm, he first solves for $F$ from the polynomial equation. In general, $n$ roots of $F$, including real and complex roots, can be found. By substituting a real $F$ and the assumed $\lambda$ into the moment equilibrium equation and from the change in the sign of the net moment for two pairs of $\left(F_{0}, \lambda_{0}\right)$ and $\left(F_{1}, \lambda_{1}\right), \lambda$ can be approximately determined by a simple interpolation scheme and the moment equilibrium equation is accordingly satisfied approximately.

Cheng's method significantly improves the convergence, but it is still possible to miss the proper solution if more than one solution exists. Let us consider the following example. By selecting $\lambda$ approximately to 0.274 here, 0.274 is $\lambda$-component in the intersection $(0.648,0.274)$, see Fig. 4, and we might obtain a solution approximate to $(0.648,0.274)$ by Cheng's method. However, the solution leads to an inadmissible force system, as seen in Fig. 6, and is thus improper. Furthermore, finding all roots of a polynomial of high degree is a typical ill-conditioned problem (Trefethen and Bau, 1997). This might be the reason that Cheng chose the double QR algorithm instead of the conventional $\mathrm{QR}$ algorithm.

To overcome the above numerical problems, in this study a robust solution procedure is developed for the rigorous slice methods. For the sake of generality, we demonstrate the application of the procedure to the discrete version of Morgenstern-Price's method. Using the procedure, one can easily find out all possible solutions and trace all distinct branches of equilibrium curves. The equilibrium curves are very informative in the GLE methods (Abramson, 1996). By observing the equilibrium curves, one can identify the equilibrium conditions to which the factor of safety is sensitive.

\section{SYSTEM OF EQUILIBRIUM EQUATIONS FOR A TYPICAL SLICE}

Suppose that the slip surface is given and the sliding mass is divided into $n$ vertical slices. A general system of forces on a typical slice is shown in Fig. 1. Any slice is always assumed to be slender enough so that the normal force on the slice base can be considered to act approximately at the base midpoint.

In this study, we will always abide by the convention of notations as follows. If some quantity can be calculated before the system of equilibrium equations is solved, a bar " " " ' is placed above the quantity, for example, the weight $\bar{w}_{\mathrm{i}}$ and the base length $\bar{b}_{\mathrm{i}}$ of slice $i$. If a quantity has no bar above it, the quantity is a variable dependent on the solution of the system of equilibrium equations, for example, the normal resisting thrust $t_{\mathrm{r}, \mathrm{i}}$ acting on slice- $i$ and the total normal force $z_{\mathrm{i}}$ on the base.

For the sake of simplicity of notations, if discussion is for a typical slice- $i$, we will omit the indices $i$ of all quanti-

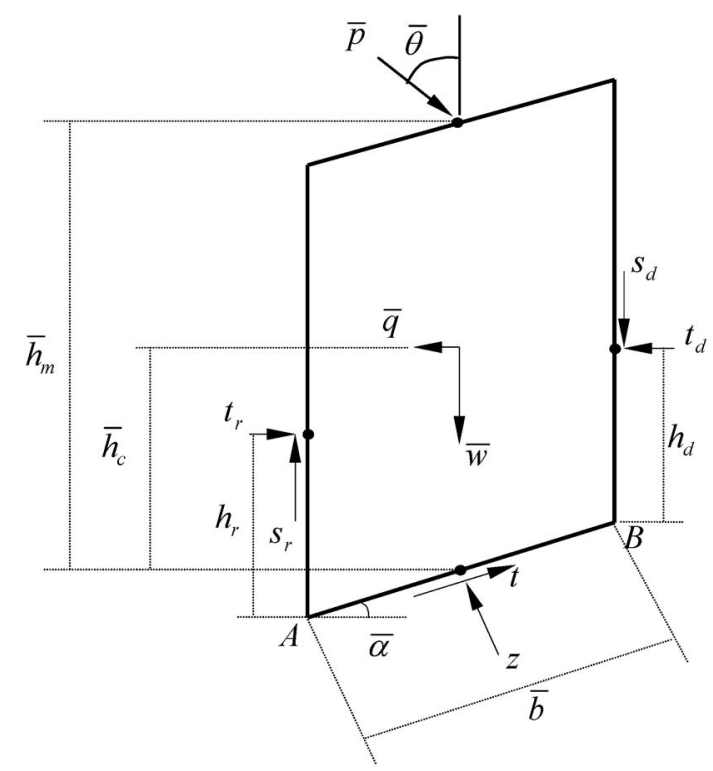

\footnotetext{
$t_{\mathrm{r}}=$ resisting horizontal thrust

$S_{\mathrm{r}}=$ resisting vertical shear

$h_{\mathrm{r}}=$ height to $t_{\mathrm{r}}$

$t_{\mathrm{d}}=$ driving horizontal thrust

$S_{\mathrm{d}}=$ driving vertical shear

$h_{\mathrm{d}}=$ height to $t_{\mathrm{d}}$

$\bar{w}=$ weight of slice

$\overline{\mathrm{q}}=$ horizontal seismic force

$\bar{h}_{\mathrm{c}}=$ height to centroid of slice

$\bar{p}=$ external surcharge

$\bar{h}_{\mathrm{m}}=$ average height of slice

$z=$ total normal force

$t=$ mobilized strength

$\bar{\alpha}=$ inclination of slice base

$\bar{b}=$ length of base
}

Fig. 1. The system of forces on a typical slice 
ties associated with slice- $i$.

Referring to Fig. 1, which shows a system of forces on a typical slice, and projecting all forces in the direction perpendicular to the base and then rearranging the equation, we have the total normal force $z$

$$
\begin{gathered}
z=s_{\mathrm{d}} \cos \bar{\alpha}-t_{\mathrm{d}} \sin \bar{\alpha}-s_{\mathrm{r}} \cos \bar{\alpha}+t_{\mathrm{r}} \sin \bar{\alpha} \\
+\bar{w} \cos \bar{\alpha}-\bar{q} \sin \bar{\alpha}+\bar{p} \cos (\bar{\theta}-\bar{\alpha})
\end{gathered}
$$

Projecting all forces in the direction of the base and then rearranging the equation, we obtain an expression for $t$

$$
\begin{gathered}
t=s_{\mathrm{d}} \sin \bar{\alpha}+t_{\mathrm{d}} \cos \bar{\alpha}-s_{\mathrm{r}} \sin \bar{\alpha}-t_{\mathrm{r}} \cos \bar{\alpha} \\
+\bar{w} \sin \bar{\alpha}+\bar{q} \cos \bar{\alpha}-\bar{p} \sin (\bar{\theta}-\bar{\alpha})
\end{gathered}
$$

Suppose that the Mohr-Coulomb condition in the limit state on the slip surface holds

$$
F t=\bar{c}_{\mathrm{b}}+\bar{f}^{\prime}\left(z-\bar{u}_{\mathrm{b}}\right)
$$

where $F$ is the safety factor, and

$$
\bar{c}_{\mathrm{b}}=\bar{c}^{\prime} \bar{b}, \quad \bar{u}_{\mathrm{b}}=\bar{u} \bar{b}
$$

$\bar{c}^{\prime}$ and $\bar{f}^{\prime}$ denote the shear strength parameters in term of effective stress of the slip surface; $\bar{u}$ is the pore pressure at the base midpoint.

Substituting Eqs. (1) and (2) into Eq. (3) and rearranging it, we have a relationship between the driving interslice force $\left(t_{\mathrm{d}}, S_{\mathrm{d}}\right)$ and the resisting interslice force $\left(t_{\mathrm{r}}, S_{\mathrm{r}}\right)$

$$
t_{\mathrm{F}} t_{\mathrm{d}}+s_{\mathrm{F}} s_{\mathrm{d}}=t_{\mathrm{F}} t_{\mathrm{r}}+s_{\mathrm{F}} s_{\mathrm{r}}+d_{\mathrm{F}}
$$

with

$$
\begin{aligned}
& t_{\mathrm{F}}=\bar{c} F+\bar{s} \bar{f}^{\prime}, \quad s_{\mathrm{F}}=\bar{s} F-\bar{c} \bar{f}^{\prime}, \quad d_{\mathrm{F}}=\bar{r}-\bar{d} F \\
& \bar{r}=\bar{c}_{\mathrm{b}}+\bar{f}^{\prime}\left(\overline{c w}-\overline{s q}+\bar{c}_{\theta} \bar{p}-\bar{u}_{\mathrm{b}}\right), \quad \bar{d}=\overline{s w}+\overline{c q}-\bar{s}_{\theta} \bar{p} \\
& \bar{c}=\cos \bar{\alpha}, \quad \bar{s}=\sin \bar{\alpha} ; \quad \bar{c}_{\theta}=\cos (\bar{\theta}-\bar{\alpha}), \quad \bar{s}_{\theta}=\sin (\bar{\theta}-\bar{\alpha})
\end{aligned}
$$

$\bar{r}$ represents the contribution of the external forces on the slice to the resisting slide; $\bar{d}$ indicates the contribution of these forces to the driving slide.

Finally, taking the base midpoint as the rotation center and formulating the moment equation, we have

$$
m_{\mathrm{d}}+\frac{\bar{b}}{2}\left(\bar{s} t_{\mathrm{d}}-\bar{c} s_{\mathrm{d}}\right)=m_{\mathrm{r}}-\frac{\bar{b}}{2}\left(\bar{s} t_{\mathrm{r}}-\bar{c} s_{\mathrm{r}}\right)+\bar{m}_{\mathrm{e}}
$$

where

$$
\bar{m}_{\mathrm{e}}=\bar{H}_{\mathrm{m}} \bar{p} \sin \bar{\theta}-\bar{H}_{\mathrm{c}} \bar{q}
$$

denotes the moment of the external forces on the slice about the base midpoint, and

$$
m_{\mathrm{d}}=h_{\mathrm{d}} t_{\mathrm{d}}, \quad m_{\mathrm{r}}=h_{\mathrm{r}} t_{\mathrm{r}}
$$

represent the moments of the normal interslice thrusts $t_{\mathrm{d}}$ and $t_{\mathrm{r}}$ about points $A$ and $B$ (see Fig. 1), respectively.

\section{RECURSION IN SLICE VARIABLES}

Up to this point, we have not introduced any assumptions into the interslice forces. In order to render the problem statically determinant, here we adopt Morgenstern-Price's assumption on the interslice forces as fol- lows

$$
S_{\mathrm{r}}=\bar{f}_{\mathrm{r}} \lambda t_{\mathrm{r}}, \quad S_{\mathrm{d}}=\bar{f}_{\mathrm{d}} \lambda t_{\mathrm{d}}
$$

where $\lambda$ is an unknown;

$$
\bar{f}_{\mathrm{r}}=f\left(\bar{x}_{\mathrm{r}}\right), \quad \bar{f}_{\mathrm{d}}=f\left(\bar{x}_{\mathrm{d}}\right)
$$

$\bar{x}_{\mathrm{r}}$ and $\bar{x}_{\mathrm{d}}$ are the abscissas of points $A$ and $B$ (see Fig. 1) respectively. $f(x)$ is a user-specified function. For Spencer's method, in particular, $f(x) \equiv 1$.

If we adopt those assumptions that have been made in other rigorous slice methods such as Janbu's method, we will have equations relating other slice variables instead of Eq. (6). However, the deduction is similar essentially to the following process. The M-P assumption is adopted based on the fact that the M-P method has better numerical properties and flexibility.

Substituting Eq. (6) into Eq. (4), we have the driving normal interslice thrust $t_{\mathrm{d}}$ expressed by the resisting normal interslice thrust $t_{\mathrm{r}}, \lambda$ and $F$

$$
t_{\mathrm{d}}=t_{\mathrm{F}}^{\prime} t_{\mathrm{r}}+d_{\mathrm{F}}^{\prime}
$$

with

$$
t_{\mathrm{F}}^{\prime}=\frac{t_{\mathrm{F}}+\bar{f}_{\mathrm{r}} \lambda s_{\mathrm{F}}}{t_{\mathrm{F}}+\bar{f}_{\mathrm{d}} \lambda s_{\mathrm{F}}}, \quad d_{\mathrm{F}}^{\prime}=\frac{d_{\mathrm{F}}}{t_{\mathrm{F}}+\bar{f}_{\mathrm{d}} \lambda s_{\mathrm{F}}}
$$

Similarly, substituting Eq. (6) into Eq. (5), we have the relationship between $m_{\mathrm{d}}$ and $m_{\mathrm{r}}$ as follows

$$
m_{\mathrm{d}}=m_{\mathrm{r}}-\frac{\bar{b}}{2}\left(\bar{s}-\bar{c} \bar{f}_{\mathrm{d}} \lambda\right) t_{\mathrm{d}}-\frac{\bar{b}}{2}\left(\bar{s}-\bar{c} \bar{f}_{\mathrm{r}} \lambda\right) t_{\mathrm{r}}+\bar{m}_{\mathrm{e}}
$$

where $t_{\mathrm{d}}$ is given by Eq. (7). For a given pair $(F, \lambda),\left(t_{\mathrm{d}}\right.$, $m_{\mathrm{d}}$ ) can be deduced from $\left(t_{\mathrm{r}}, m_{\mathrm{r}}\right)$ by using Eqs. (7) and (8).

However, we must stress the fact that Eq. (7) holds only if the condition

$$
\delta_{\mathrm{d}}+\phi_{\mathrm{F}}-\bar{\alpha} \neq \frac{\pi}{2}
$$

is satisfied. Where $\delta_{\mathrm{d}}=t g^{-1}\left(\lambda \bar{f}_{\mathrm{d}}\right)$ is the angle of the driving interslice force vector $\left(t_{\mathrm{d}}, s_{\mathrm{d}}\right)$ with the axis- $x$; and $\phi_{\mathrm{F}}=$ $\operatorname{tg}^{-1}\left(\bar{f}^{\prime} / F\right)$ represents the limit frictional angle.

In fact, if inequality Eq. (9) becomes an equality, the denominator of $t_{\mathrm{F}}^{\prime}$ and $d_{\mathrm{F}}^{\prime}$ in Eq. (7a), $t_{\mathrm{F}}+\bar{f}_{\mathrm{d}} \lambda s_{\mathrm{F}}$, can be proved to equal zero. This would render Eq. (7) meaningless.

If a given pair $(F, \lambda)$ leads to a slice satisfying $\delta_{\mathrm{d}}+\phi_{\mathrm{F}}-$ $\bar{\alpha}=\pi / 2$, Baker (1980) proved that the slice cannot be in equilibrium because the force polygon of the slice cannot be closed. Therefore, such a pair $(F, \lambda)$ cannot be a solution of the problem. This is a very important conclusion that will be used in the following.

In the subsequent analysis, we will refer numerically to a pair $(F, \lambda)$ leading to some slices satisfying $\mid \delta_{\mathrm{d}}+\phi_{\mathrm{F}}-\bar{\alpha}$ $-90^{\circ} \mid<2^{\circ}$ as taboo; whereas otherwise the pair $(F, \lambda)$ is feasible. Accordingly, a set of all taboo pairs is referred to as a taboo domain, for example, the hatched domain in Fig. 4.

Now let us consider the whole slices from toe to top. If a feasible pair $(F, \lambda)$ is given, beginning with slice-1 (at 
the toe) with the given vector $\overline{\boldsymbol{g}}_{\mathrm{r}, 1}=\left(\bar{t}_{\mathrm{r}, 1}, \bar{m}_{\mathrm{r}, 1}\right)^{\mathrm{T}}$ (being a null vector usually) and using Eqs. (7) and (8) in a recursive manner, slice-by-slice, one will obtain a vector $\boldsymbol{g}_{\mathrm{d}, \mathrm{n}}=$ $\left(t_{\mathrm{d}, \mathrm{n}}, m_{\mathrm{d}, \mathrm{n}}\right)^{\mathrm{T}}$ associated with slice- $n$ (at the top). Therefore, such a process actually defines a nonlinear map: $M: R_{+}^{2} \rightarrow R^{2}$,

$$
M(\lambda, F)=g_{\mathrm{d}, \mathrm{n}}
$$

with $R_{+}^{2}=\left\{(x, y) \in R^{2} \mid x>0, y>0\right\}$. Our task is to find a $(\lambda, F) \in R_{+}^{2}$ such that the vector $g_{\mathrm{d}, \mathrm{n}}=\left(t_{\mathrm{d}, \mathrm{n}}, m_{\mathrm{d}, \mathrm{n}}\right)^{\mathrm{T}}$ decided by map $\boldsymbol{M}$ equals the given vector $\overline{\boldsymbol{g}}_{\mathrm{d}, \mathrm{n}}=\left(\bar{t}_{\mathrm{d}, \mathrm{n}}, \overline{\boldsymbol{m}}_{\mathrm{d}, \mathrm{n}}\right)^{\mathrm{T}}$ on slice- $n$, which is also a null vector unless a tension crack filled with water is set at the top of the sliding mass. In other words, one has to solve the system of nonlinear equations in $\lambda$ and $F$

$$
\boldsymbol{g}(F, \lambda) \equiv \boldsymbol{g}_{\mathrm{d}, \mathrm{n}}(F, \lambda)-\overline{\boldsymbol{g}}_{\mathrm{d}, \mathrm{n}}=0
$$

where $g(F, \lambda) \equiv\left(g_{1}, g_{2}\right)^{\mathrm{T}}$ is a 2-dimensional vector-valued function with components:

$$
\begin{aligned}
& g_{1}(F, \lambda) \equiv t_{\mathrm{d}, \mathrm{n}}(F, \lambda)-\bar{t}_{\mathrm{d}, 1} \\
& g_{2}(F, \lambda) \equiv m_{\mathrm{d}, \mathrm{n}}(F, \lambda)-\bar{m}_{\mathrm{d}, 1}
\end{aligned}
$$

\section{PROCEDURE OF COMPLETED-LABELED CELLS}

We first give the solution procedure for the system (11) and then justify it:

1) Give tolerances of $F$ and $\lambda: \varepsilon_{\mathrm{F}}$ and $\varepsilon_{\lambda}$. Usually, a relative error of $1 \%$ is a satisfactory precision for $F$. But, in this study, we take more stringent error control and let $\varepsilon_{\lambda}=\varepsilon_{\mathrm{F}}=10^{-3}$.

2) Give two intervals for $F$ and $\lambda$ respectively, $\left[F_{0}, F_{1}\right]$ and $\left[\lambda_{0}, \lambda_{1}\right]$. This step is not difficult even for those who have little experience in the stability analysis of slopes. For example, in order to set interval $\left[F_{0}, F_{1}\right]$ for $F$, a non-rigorous method can be used to calculate an approximate value of $F$, say $F^{\prime}$. Then, set $\left[F_{0}, F_{1}\right]$ $=\left[0.8 F^{\prime}, 1.2 F^{\prime}\right]$. As for interval $\left[\lambda_{0}, \lambda_{1}\right]$ of $\lambda$, one can learn from Corps of engineer's method (Abramson et al., 1996) and let $\left[\lambda_{0}, \lambda_{1}\right]=[\operatorname{tg}(0.1 \theta), \operatorname{tg}(1.4 \theta)]$ with $\theta$ being the average inclination of the slope in consideration.

3) Dividing intervals $\left[F_{0}, F_{1}\right]$ and $\left[\lambda_{0}, \lambda_{1}\right]$ into $n_{\mathrm{F}}$ and $n_{\lambda}$ shorter intervals respectively, we have $n_{\mathrm{F}} \times n_{\lambda}$ cells within a rectangle with a diagonal of $\left[F_{0}, \lambda_{0}\right]-\left[F_{1}, \lambda_{1}\right]$ as shown in Fig. 2. Here,

$$
n_{\mathrm{F}}=\operatorname{int} \frac{F_{1}-F_{0}}{\varepsilon_{\mathrm{F}}}, \quad n_{\lambda}=\operatorname{int} \frac{\lambda_{1}-\lambda_{0}}{\varepsilon_{\lambda}}
$$

In this way, we obtain $\left(n_{\mathrm{F}}+1\right) \times\left(n_{\lambda}+1\right)$ nodes. A typical node coded $(i, j)$ has the coordinate $\left(F_{\mathrm{i}}, \lambda_{\mathrm{j}}\right)$ as follows

$$
\begin{aligned}
& F_{\mathrm{i}}=F_{\mathrm{i}-1}+\varepsilon_{\mathrm{F}}, \quad j=1, \cdots, n_{\mathrm{F}}+1 \\
& \lambda_{\mathrm{j}}=\lambda_{\mathrm{j}-1}+\varepsilon_{\lambda}, \quad i=1, \cdots, n_{\lambda}+1
\end{aligned}
$$

4) Label all nodes in Fig. 2. Take a typical node $(i, j)$ as an example. First, $g_{1}\left(F_{\mathrm{i}}, \lambda_{\mathrm{j}}\right)$ and $g_{2}\left(F_{\mathrm{i}}, \lambda_{\mathrm{j}}\right)$ are calculated; then, an integer $L(i, j)$, called label, is assigned to node $(i, j)$, which is defined by

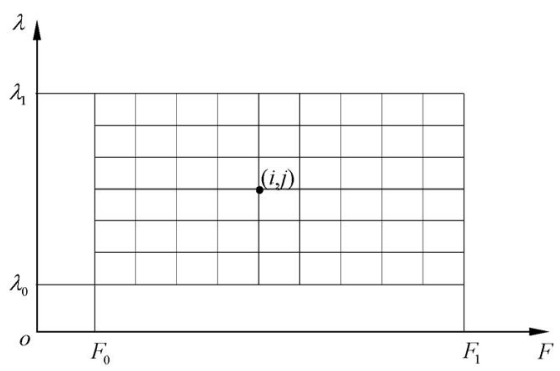

Fig. 2. Division of the potential scope of solution

$$
L(i, j)=\left\{\begin{array}{l}
0, \text { if } g_{1} \leq 0 \text { and } g_{2} \leq 0 \\
1, \text { if } g_{1}>0 \\
2, \text { if } g_{1} \leq 0 \text { and } g_{2}>0 \\
3, \text { if }\left(F_{\mathrm{i}}, \lambda_{\mathrm{j}}\right) \text { is taboo }
\end{array}\right.
$$

5) Indentify all Completely-Labelled Cells. One can check all cells in Fig. 2 in row order and from bottom to top. Suppose a cell with four nodes: $(i, j),(i+1, j)$, $(i+1, j+1)$ and $(i, j+1)$ is in consideration. The set of labels of the four nodes of the cell, denoted by $S_{\mathrm{L}}$, must be a subset of the integer set $\{0,1,2,3\}$. Two cases must be considered.

Case-I: none of the four nodes is taboo. In this case, $S_{\mathrm{L}}$ must be a subset of the integer set $\{0,1,2\}$. If $S_{\mathrm{L}}$ is a proper subset of set $\{0,1,2\}$, go to the next cell. Otherwise, $S_{\mathrm{L}}=\{0,1,2\}$, we refer to the cell as a Completely-Labelled Cell (CLC), and regard its center coordinate, written as $\left(F_{\mathrm{i}}^{\mathrm{c}}, \lambda_{\mathrm{j}}^{\mathrm{c}}\right)$, as a possible solution of the problem.

Case-II: at least one of the four nodes is taboo, i.e., label " 3 "' is an element of $S_{\mathrm{L}}$. In this case, the cell cannot contain a solution. So, we label the cell as a taboo cell and then fetch the next cell to check.

6) Identify the true solution from all possible solutions $\left(F_{\mathrm{i}}^{\mathrm{c}}, \lambda_{\mathrm{j}}^{\mathrm{c}}\right)$ found in step 5 . This can be done through checking which of the possible solutions leads to a statically admissible system of slice forces. If none of such solutions exists, the slip surface in consideration might be far from the critical slip surface.

The above solution procedure is based on the following theorem. In this discussion, we suppose that a vectorvalued function $f: D \subset R^{2} \rightarrow R^{2}$ is continuous on a close domain $D$

$$
\boldsymbol{f}(\boldsymbol{x})=\left(\begin{array}{l}
f_{1}\left(x_{1}, x_{2}\right) \\
f_{2}\left(x_{1}, x_{2}\right)
\end{array}\right), \quad \boldsymbol{x} \in D
$$

Since $f(x)$ is continuous on the close domain $D, f(x)$ is also uniformly continuous on $D$ according to Cantor's theorem in mathematical analysis. This indicates that for any given positive $\varepsilon$ there exists a positive $\delta$ depending solely on $\varepsilon$ such that for any two points $x, y \in D$ with $\| x-$ $y \|<\delta$ the inequality $\|f(x)-f(x)\|<\varepsilon / 2$ is implied. Here, $\|\boldsymbol{x}\|=\max \left(\left|x_{1}\right|,\left|x_{2}\right|\right)$ with $\boldsymbol{x}=\left(x_{1}, x_{2}\right)$.

While partitioning $D$, we always divide it into a number of quadrilateral cells that are regular enough in shape, and obtain a partition $P$.

We also give all nodes in partition $P$ a label in such a 
way that node- $i$ with coordinate $\boldsymbol{x}^{i}=\left(x_{1}^{i}, x_{2}^{i}\right)$ has the label defined as follows

$$
L(i)=\left\{\begin{array}{l}
1, \text { if } f_{1}\left(x^{i}\right)>0 \\
2, \text { if } f_{1}\left(x^{i}\right) \leq 0 \text { and } f_{2}\left(x^{i}\right)>0 \\
0, \text { if } f_{1}\left(x^{i}\right) \leq 0 \text { and } f_{2}\left(x^{i}\right) \leq 0
\end{array}\right.
$$

We denote by $\operatorname{dia}(P)$ the longest diagonal of all these cells in partition $P$. Now we can conclude as follows. If $D$ has a partition $P$ with $\operatorname{dia}(P)<\delta$ and a CLC, denoted by $\sigma$, as defined in step 5, we have $\|\boldsymbol{f}(\boldsymbol{x})\|<\varepsilon$ for any $\boldsymbol{x} \in \sigma$.

Proof. Since $\sigma$ is a CLC, it has at least three nodes, written as $\boldsymbol{u}^{0}, \boldsymbol{u}^{1}$ and $\boldsymbol{u}^{2}$, whose labels are 0,1 , and 2 , respectively. According to the label definition Eq. (13), we have

$$
f_{1}\left(\boldsymbol{u}^{1}\right)>0, \quad f_{2}\left(\boldsymbol{u}^{2}\right)>0
$$

and

$$
f_{1}\left(\boldsymbol{u}^{0}\right) \leq 0, \quad f_{2}\left(\boldsymbol{u}^{2}\right) \leq 0
$$

For any point $\boldsymbol{x} \in \Delta \boldsymbol{u}^{0} \boldsymbol{u}^{1} \boldsymbol{u}^{2}$, where $\Delta \boldsymbol{u}^{0} \boldsymbol{u}^{1} \boldsymbol{u}^{2}$ represents the triangle with $\boldsymbol{u}^{0}, \boldsymbol{u}^{1}$ and $\boldsymbol{u}^{2}$ as its vertices, we first prove $\left|f_{1}(x)\right|<\varepsilon / 2$.

$\boldsymbol{x} \in \Delta \boldsymbol{u}^{0} \boldsymbol{u}^{1} \boldsymbol{u}^{2}$ implies $\left\|\boldsymbol{u}^{1}-\boldsymbol{x}\right\| \leq \operatorname{dia}(P)<\delta$ and $\mid f_{1}\left(\boldsymbol{u}^{1}\right)-$ $f_{1}(\boldsymbol{x}) \mid<\varepsilon / 2$. The latter leads to

$$
f_{1}(x)-f_{1}\left(u^{1}\right) \geq-\left|f_{1}\left(u^{1}\right)-f_{1}(x)\right|>-\frac{\varepsilon}{2}
$$

From $f_{1}\left(\boldsymbol{u}^{1}\right)>0$ and the above inequality, we have $f_{1}(\boldsymbol{x})>$ $-(\varepsilon / 2)$.

$\boldsymbol{x} \in \Delta \boldsymbol{u}^{0} \boldsymbol{u}^{1} \boldsymbol{u}^{2}$ implies $\left\|\boldsymbol{u}^{0}-\boldsymbol{x}\right\|<\delta$ and $\left|f_{1}\left(\boldsymbol{u}^{0}\right)-f_{1}(\boldsymbol{x})\right|$ $<\varepsilon / 2$. Again, the latter leads to

$$
f_{1}(x)-f_{1}\left(u^{0}\right) \leq\left|f_{1}(x)-f_{1}\left(u^{0}\right)\right|<\frac{\varepsilon}{2}
$$

From $f_{1}\left(\boldsymbol{u}^{0}\right) \leq 0$ and the above inequality, $f_{1}(\boldsymbol{x})<\varepsilon / 2$ is followed. Therefore, we have $\left|f_{1}(\boldsymbol{x})\right|<\varepsilon / 2$.

A similar deduction applies to the proof of $\mid f_{2}$ $(\boldsymbol{x}) \mid<\varepsilon / 2$ as long as all $f_{1}(\cdot)$ and $\boldsymbol{u}^{1}$ are replaced with $f_{2}$ (·) and $\boldsymbol{u}^{2}$, respectively. We thus have $\|f(x)\|<\varepsilon / 2$ for any $\boldsymbol{x} \in \Delta \boldsymbol{u}^{0} \boldsymbol{u}^{1} \boldsymbol{u}^{2}$.

Now suppose that $y \in \sigma$. Since $\boldsymbol{x} \in \Delta \boldsymbol{u}^{0} \boldsymbol{u}^{1} \boldsymbol{u}^{2} \subset \sigma$ implies $\|y-x\|<\delta$ and $\|f(y)-f(x)\|<\varepsilon / 2$, we have

$$
\|f(y)\| \leq\|f(y)-f(x)\|+\|f(x)\|<\varepsilon
$$

Up to this point, we have proved the conclusion.

However, there is a problem when the above theorem is used to solve system (11). As discussed in the foregoing analysis, there might be some taboo cells in the domain shown in Fig. 2 where $g(F, \lambda)$ has no definition, whereas the theorem demands a continuous $g(F, \lambda)$. But this would not pose problems because $g(F, \lambda)$ is still continuous on the domain from which those taboo cells are removed.

\section{TWO ILLUSTRATIVE EXAMPLES}

Example 1. This example was presented by Chugh (1981), who adopted Spencer's method to solve it by Newton's method but failed because a system of non-

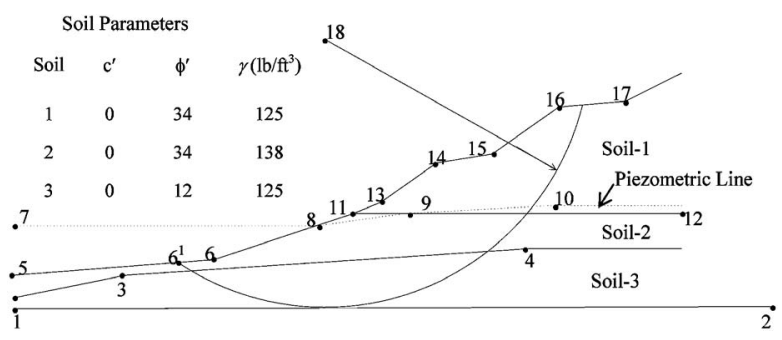

Fig. 3. Geometrical and soil parameters of Example 1

Table 1. Coordinates and surface tractions of relevant nodes

\begin{tabular}{r|r|r|r|r|r|r|l}
\hline Node & \multicolumn{1}{|c|}{$x$} & \multicolumn{1}{c|}{$y$} & Traction & Node & \multicolumn{1}{c|}{$x$} & \multicolumn{1}{c|}{$y$} & Traction \\
\hline 1 & 0.0 & 870.0 & & 10 & 480.0 & 962.0 & \\
\hline 2 & 680.0 & 872.0 & & 11 & 305.0 & 955.0 & \\
\hline 3 & 100.0 & 900.0 & & 12 & 600.0 & 955.0 & \\
\hline 4 & 465.0 & 924.0 & & 13 & 330.0 & 965.0 & \\
\hline 5 & 0.0 & 900.0 & & 14 & 380.0 & 1000.0 & \\
\hline 6 & 147.8 & 911.5 & 2028.0 & 15 & 430.0 & 1008.0 & \\
\hline 6 & 180.0 & 914.0 & 1872.0 & 16 & 490.0 & 1050.0 & \\
\hline 7 & 0.0 & 944.0 & & 17 & 550.0 & 1055.0 & \\
\hline 8 & 271.5 & 944.0 & 0.0 & 18 & 280.0 & 1110.0 & \\
\hline 9 & 350.0 & 955.0 & & & & & \\
\hline
\end{tabular}

Note: Node $6^{\prime}$ is not a characteristic point but the intersection point of the failure circle and line 5-6

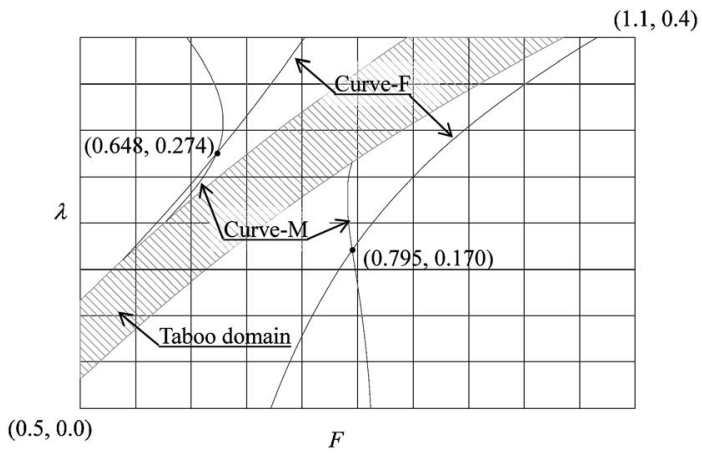

Fig. 4. Two distinct branches of equilibrium curves

linear equations similar to system Eq. (11) has two solutions that are quite close to each other. That urged him to develop the numerically-graphical solution procedure that has been evaluated previously.

Figure 3 shows the conditions of the slope. In order that other researchers repeat the example easily, the data involved are duplicated from the original problem and the English system is retained. Table 1 lists the coordinates and surface tractions (caused by the river water) of the points relevant to the analysis.

We still use Spencer's method but solve the system of equations by the proposed solution procedure. The initial interval for $F$ is $[0.5,1.4]$ and for $\lambda$ is $[0.0,0.40]$. Two 


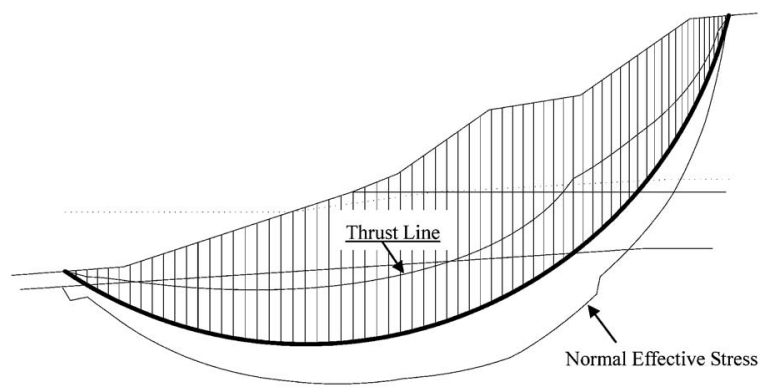

Fig. 5. Calculated response for the first possible solution

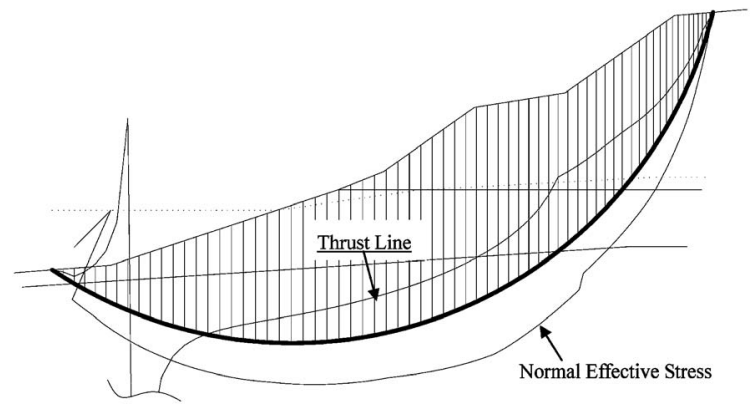

Fig. 6. Calculated response for the second possible solution

distinct branches of equilibrium curves are tracked and shown in Fig. 4, with two distinct intersections: $(F, \lambda)^{1}=$ $(0.795,0.170)$ and $(F, \lambda)^{2}=(0.648,0.274)$, very close to Chugh's two solutions: $(F, \lambda)^{\mathrm{C} 1}=(0.796,0.170)$ and $(F$, $\lambda)^{\mathrm{C} 2}=(0.652,0.273)$. Because of the presence of a taboo domain, marked with hatching lines in Fig. 4, the two branches of equilibrium curves are isolated from each other by the taboo domain and three equilibrium curves cannot reach the boundary of the initial rectangle with diagonal $(0.5,0.0)-(1.4,0.4)$ but terminate near the boundary of the taboo domain.

Figure 4 tells us that only if the initial value $\left(F_{0}, \lambda_{0}\right)$ is in the bottom-right domain the right intersection $(F, \lambda)^{1}$ might be sought by the conventional iterative solution procedures. Otherwise, one of the two cases will occur: 1$)$ if $\left(F_{0}, \lambda_{0}\right)$ is in the upper-left domain, the intersection $(F$, $\lambda)^{2}$, an improper solution, will be sought; 2$)$ if $\left(F_{0}, \lambda_{0}\right)$ is in the taboo domain, the solution will break down no matter what solvers are used. Even if the initial point $\left(F_{0}\right.$, $\left.\lambda_{0}\right)$ is not in the taboo domain, some conventional methods might fail to reach any intersection (Cheng et al., 2008). For example, Chugh (1981) tried Newton's method to solve the example but none of the intersections was sought.

Figures 5 and 6 illustrate the results from the two solutions respectively. It can be seen that only the first intersection $(F, \lambda)^{1}$ is the solution to the problem.

Example 2. This example is Problem 3b issued by Austrian Computer Aided Design Association (ACADS) (Donald and Giam, 1992) and is used to examine the capability of the proposed procedure to treat non-circular slip surfaces. The strength parameters of the soils and the

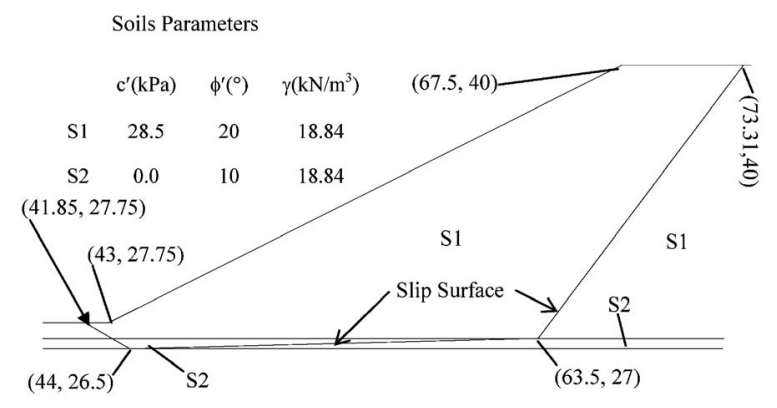

Fig. 7. Geometrical and soil parameters of Example 2

geometry of the slope are shown in Fig. 7.

Let $\left[F_{0}, F_{1}\right]=[1.0,1.7]$ and $\left[\lambda_{0}, \lambda_{1}\right]=[0.0,0.7]$. A constant and a half-sine function are used to describe the variation of the angle of the interslice forces, respectively. For both cases, only one CLC is obtained. The results are listed as follows

$\begin{array}{lcc}\text { Function } & F & \lambda \\ \text { Constant (Spencer) } & 1.275 & 0.196 \\ \text { Half-sine } & 1.260 & 0.233\end{array}$

Among all test examples issued by ACADS, this example displayed the most random scatter: the safety factors calculated by different analysts ranged from 1.197 to 1.450 (Donald and Giam, 1992). These reports showed that to some degree it was sensitive to the choice of function $f(x)$ in the M-P method even if the rigorous methods were used. Later on, Krahn (2003) also demonstrated that in situations where the slip surface consists of multiple straight lines and the slip mass was not homogeneous the form of $f(x)$ in the M-P method might have a very significant effect on the solution.

However, it seems that the results from this example do not support the above conclusion. Instead, it accords with the statement by Duncan (1996), who claimed that the maximum difference between factors of safety calculated by the rigorous methods is about $12 \%$, usually less. For this example, the difference between Spencer's method (1.275) and the M-P's method (half-sine, 1.260) is less than $1.12 \%$.

The calculated factor of safety, 1.260, corresponding to the half-sine function agrees well with 1.261 offered by Fredlund who also selected the same function in the M-P method.

Figure 8 illustrates the equilibrium curves for Spencer's method and the M-P method (half-sine) respectively. In both cases, no taboo domain exists. Therefore, an arbitrary initial value for $(F, \lambda)$ should successfully lead to finding the intersection.

Shown in Fig. 9 are the results from Spencer's method and the M-P method (half-sine). In both cases, parts of the thrust lines near the top of the slope lie outside the slip mass. This means that some interslice thrusts are tensile. If the slip mass were rock mass with large enough tension strength, the results would be acceptable. If not, a tension crack should be set somewhere near the top of the sliding mass so that no interslice thrust is tensile. 

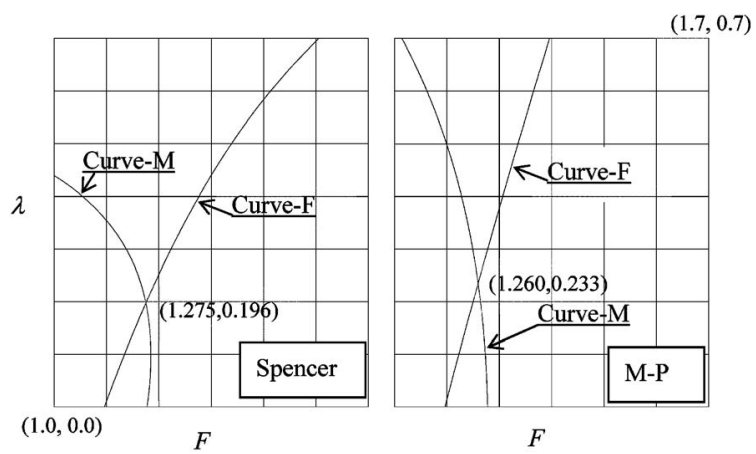

Fig. 8. Equilibrium curves for Spencer's method and M-P's method

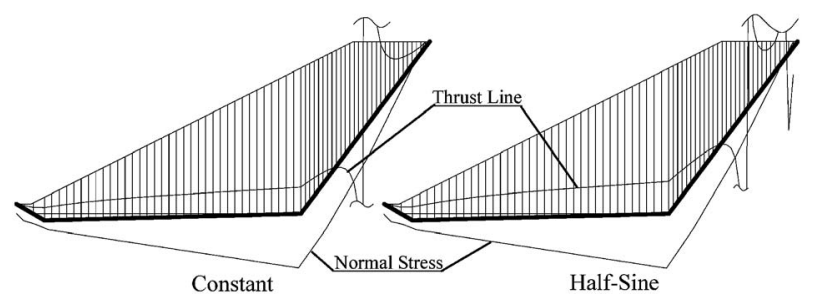

Fig. 9. Results corresponding to two functions

\section{COMMENTS AND CONCLUSIONS}

From the above discussion, the proposed solution procedure is characterized by the following items:

1) Whether the solution to a problem in consideration exists within a specified scope can be detected during the process of finding a solution, and all the solutions can be sought as long as they exist. The non-convergence adhering to the rigorous slice methods has thus been overcome.

2) No information on derivatives of the functions is needed; therefore, it is fit to solve those systems of nonlinear equations that are induced by a new slice method that will probably be developed in future, where the functions may be merely continuous but not smooth.

3) Because only the signs (instead of values) of the functions are involved, the demand for memory storage is low.

4) The precision of solution is easy to control.

5) Distinct domains with different characteristics in plane- $(F, \lambda)$ can be identified, from which one can know how to select a feasible value for $(F, \lambda)$ so as to reach a solution.

To implement the solution procedure, it suffices to specify a domain in plane- $(F, \lambda)$ that contains the solutions.

Compared with those conventional solution procedures, the proposed procedure might be less efficient. However, it should not be a big problem given current available computer processing capabilities. Using a normal Pentium PC with a $3.0 \mathrm{GHz}$ processor, it only takes about $95 \mathrm{~s}$ to solve an example in the above, including the generation and output of all graphical data. Besides, this is achieved under a fine division (70 slices) and a high precision $\left(10^{-3}\right)$ for both $F$ and $\lambda$. If these conditions are relaxed as usual, for example, a division of 30 slices and a precision of $10^{-2}$ for $F$ and $\lambda$, the computation can be completed in a few seconds.

If all the conventional solution procedures fail to converge, the proposed procedure deserves being used.

\section{ACKNOWLEDGEMENTS}

The authors gratefully acknowledge the financial support provided by the "Research Project for Protection of High Cut Slopes in Three Gorges' Reservoir under Contract Number 2008SXG01-2"'.

\section{REFERENCES}

1) Abramson, L. W., Lee, T. S., Sharma, S. and Boyce, G. M. (1996): Slope Stability and Stabilization Methods, Wiley, New York.

2) Baker, R. (1980): Determination of the critical slip surface in slope stability computation, International Journal for Numerical and Analytical Methods in Geomechanics, 4, 333-359.

3) Baker, R. (2005): Variational slope stability analysis of materials with non-linear failure criterion, Electronic Journal of Geotechnical Engineering, 10(Bundle A), 1-22.

4) Cheng, Y. M. (2003): Location of critical failure surface and some further studies on slope stability analysis, Computers \& Geotechnics, 30, 255-267.

5) Cheng, Y. M. and Zhu, L. J. (2004): Unified formulation for two dimensional slope stability analysis and limitations in factor of safety determination, Soils and Foundations, 44, 121-127.

6) Cheng, Y. M., Lansivaara, T. and Siu, J. (2008): Impact of convergence on slope stability analysis and design, Computers \& Geotechnics, 35, 105-113.

7) Chugh, A. K. (1981): Multiplicity of numerical solutions for slope stability problems, International Journal for Numerical and Analytical Methods in Geomechanics, 5, 313-322.

8) Donald, I. B. and Giam, P. (1992): The ACADS slope stability programs review, Proc. 6th International Symposium on Landslides, $\mathbf{3}$, 1665-1670.

9) Duncan, J. M. (1996): State of the Art: Limit equilibrium and finite element analysis of slopes, Journal of Geotechnical Engineering, ASCE, 122, 577-596.

10) Husein, A. I., Hassan, W. F. and Sarma, S. K. (2001): Global search method for locating general slip surface using Monte Carlo techniques, Journal of Geotechnical Engineering, ASCE, 127, 688-698.

11) Krahn, J. (2003): The 2001 R. M. Hardy Lecture; The limits of limit equilibrium analysis, Canadian Geotechnical Journal, 40, 643-660.

12) Trefethen, L. N. and Bau, D. (1997): Numerical Linear Algebra, SIAM, Philadelphia.

13) Zheng, H., Liu, D. F. and Li, C. G. (2005): Slope stability analysis based on elasto-plastic finite element method, International Journal Numerical Methods in Engineering, 64, 1871-1888.

14) Zheng, H., Tham, L. G. and Liu, D. F. (2005): On two definitions of the factor of safety commonly used in the finite element slope stability analysis, Computers \& Geotechnics, 33, 188-195.

15) Zhu, D. Y., Lee, C. F. and Qian, Q. H. (2005): A concise algorithm for computing the factor of safety using the Morgenstern-Price method, Canadian Geotechnical Journal, 42, 272-278. 